\title{
Suicidabilidad y apoyo social percibido en estudiantes universitarios con enfermedades crónicas no transmisibles
}

\section{Suicidality and perceived social support in university students with chronic non-communicable diseases}

\author{
Tamara Otzen \\ Universidad de La Frontera, Temuco, Chile \\ Facultad de Ciencias de la Salud, Universidad de Tarapacá, Arica, Chile. \\ Natalia Fuentes \\ Gustavo Wetzel \\ Constanza Henríquez \\ Carrera de Psicología, Universidad Autónoma de Chile. \\ Zayra Antúnez \\ Centro de Salud Universitario, Universidad Austral de Chile, Valdivia, Chile. \\ Instituto de Estudios Psicológicos, Universidad Austral de Chile, Valdivia, Chile \\ Tamara Melnik \\ Universidade Federal de São Paulo, São Paulo SP, Brasil \\ Rec (26 de Diciembre de 2019) Acept (17 de Marzo de 2020)
}

\begin{abstract}
Resumen
El objetivo fue identificar las asociaciones entre apoyo social percibido, riesgo suicida y presencia de enfermedad crónica no transmisible en estudiantes universitarios. Utilizando un diseño de casos y controles, con 41 estudiantes universitarios por grupo. La mediana de edad fue de 22 años $(R=9)$, un $81,8 \%$ eran mujeres, el 26,8\% de los sujetos tenía Asma y el 26,8\% Hipotiroidismo, y el 63,6\% pertenecía a la Facultad de Educación. No se comprobó la asociación entre enfermedad crónica, apoyo social, ni tampoco con riesgo suicida. Hay asociación indirecta entre riesgo suicida y apoyo social, no así cuando se evalúa el intento de suicidio previo. El nivel de riesgo suicida y apoyo social se asocian independiente de la presencia de enfermedad crónica. Esperamos que nuestros resultados permitan fomentar el apoyo social como una herramienta fundamental para la prevención de la suicidabilidad, especialmente en sujetos con enfermedad crónica.

Palabras Claves: Suicidio, Enfermedad Crónica, Apoyo Social, Estudiante, Chile
\end{abstract}

Correspondencia: Tamara Otzen, Universidad de La Frontera, Avenida Francisco Salazar 01145, Temuco, Chile, +56452596917 , tamara.otzen@ufrontera.cl.

Agradecimientos: Artículo financiado por la Universidad de La Frontera, Proyecto IAF17-0003 


\begin{abstract}
The objective was to identify associations between perceived social support, suicidal risk and the presence of chronic or communicable disease in university students. Using a case-control design, consisting of 41 subjects in each group. The median age was 22 years $(\mathrm{R}=9), 81,8 \%$ were women, $26,8 \%$ of the subjects had asthma and $26,8 \%$ had hypothyroidism, $63,6 \%$ were students of the faculties of Education. The association between chronic disease and social support was not proven, nor was there a suicidal risk. There is an indirect association between suicide risk and social support, not so when assessing the previous suicide attempt. The levels of suicide risk and social support are associated, independent of an existing chronic disease. We are hopeful that our results will be effective in promoting social support as a basic tool for suicide prevention, especially in subjects with chronic disease.

Keywords: Suicide, Chronic Disease, Social Support, Students, Chile
\end{abstract}

\title{
Introducción
}

Las enfermedades crónicas no transmisibles (ECNT) son un importante problema de salud pública, ya que son enfermedades de larga duración, por lo general de progresión lenta y que implican algún grado de limitación en la vida cotidiana (Yeo \& Sawyer, 2005; OMS, 2015a). En la actualidad, constituyen un importante desafío que debió asumir la salud pública en el último medio siglo (Serrano \& González, 2002), puesto que implican elevados costos personales, sociales y económicos para la sociedad (Azevedo, Costa-Pereira, Mendonça, Dias \& Castro-Lopes, 2016). Las ECNT influyen en la escasez de mano de obra y provocan un aumento del gasto del gobierno en servicios de salud (Schofield et al., 2016).

En Chile, al igual que en la mayoría del mundo (OMS, 2015a), las ECNT son la principal causa de muerte, siendo las más prevalentes: el cáncer, la diabetes, la hipertensión arterial, el hipotiroidismo, el asma y la resistencia a la insulina (DEIS, 2016).

Por otra parte, la calidad de vida y el estado funcional de los enfermos crónicos es limitado (López, Beltrán, Moreno \& Estrada, 2007), generando grave impacto psicológico, repercutiendo en el área personal y social del individuo (Garay-Sevilla, 2005; Vega Angarita \& González Escobar, 2009). Las consecuencias psicológicas de manifestar una ECNT implican un esfuerzo de adaptación y la manifestación de estados emocionales dominados por la aprehensión, preocupación por la salud y pronóstico, además de incertidumbre, miedo, ansiedad, depresión (Vinaccia \& Orozco, 2005; Navarro, 2007), problemas de adaptación a la enfermedad e incluso un constante miedo a la muerte (López, 2007). Específicamente, los antecedentes indican que las personas con enfermedades crónicas, tendrían una incidencia de depresión tres veces superior a la de la población general, lo que se asocia a un aumento de ideación y riesgo suicida (Oblitas, 2006; López, 2007; Lutz, Morton, Turiano \& Fiske, 2016).

A nivel familiar, se ven alteradas las relaciones intrafamiliares y de pareja, puesto que los enfermos crónicos generalmente requieren del apoyo y cuidados de los cercanos, lo que puede generar estrés y malestar en el cuidador principal y demás miembros de la familia (Helgeson, Jakubiak, Van Vleet \& Zajdel, 2018; Durán, 2004). En el ámbito laboral y económico, una de las principales consecuencias es el ausentismo laboral (Díaz, Marulanda \& Sáenz, 2009). A nivel social, los sujetos que padecen enfermedades crónicas tienden a aislarse, lo que ha hecho considerar que el apoyo social que reciben de otros es un aspecto primordial en el mejoramiento de la calidad de vida de las personas con problemas de salud crónicos (Díaz et al., 2009).

El apoyo social que los enfermos crónicos reciban puede protegerles de los efectos patogénicos que desencadenan las situaciones estresantes, incluso cuando las enfermedades son intensas o prolongadas (Barra, 2004). Asimismo, el apoyo social tendría consecuencias positivas en el desarrollo de mecanismos de afrontamiento, disminución de ansiedad, depresión y aislamiento, potenciando la salud en general (Kimberly \& Serovich, 1999; Pérez \& Martín, 2006; Vega Angarita \& González Escobar, 2009; Adelman, Tmandova, Delgado, Dion \& Lachs, 2014) e influyendo en la disminución de la mortalidad de sujetos con enfermedades crónicas (Tunbridge et al., 1977).

El apoyo social de familiares y amigos genera un mayor impacto en los sujetos, ya que está constituido por las personas más significativas e importantes (Feldman, Goncalves, Chacon-Puignau, Zaragoza, Bagés \& De Pablo, 2008). Generalmente, es la familia la principal fuente de apoyo instrumental, afectivo y emocional 
con que cuenta el paciente crónico para lograr un afrontamiento efectivo a los problemas que se originan con la enfermedad (Ponce, Velásquez, Márquez, López \& Bellido, 2009). No obstante, las dificultades familiares también podrían provocar un aumento en los niveles de estrés, de síntomas depresivos y en la ideación suicida, ya que un clima de tensión, hostilidad o violencia en la familia es un factor que potencia el riesgo suicida, al igual que la falta de apoyo social, de redes y la sensación de aislamiento en lo referente a la familia, parientes y amigos (Rivera, 2000; Feldman et al., 2008; MINSAL, 2013; Law et al., 2014).

En referencia al suicidio, la evidencia indica que el estrés causado por conflictos interpersonales puede preceder al intento suicida (Hagedorn \& Omar, 2002), siendo el apoyo social un factor moderador entre los síntomas depresivos y la ideación suicida, disminuyendo la relación entre estas variables (Lamis, Ballard, May \& Dvorak, 2016). Al respecto, la familia puede ser un elemento protector para la conducta suicida, principalmente en adolescentes, ya que cuando existe mayor apoyo social reflejado en unión, apoyo y expresión de las emociones con la familia se presentan menos intentos suicidas (Rivera \& Andrade, 2006).

En estudiantes universitarios, estudios como el de Sánchez, Cáceres \& Gómez (2002) muestran que los trastornos depresivos y el maltrato sufrido en la infancia se relaciona con la ideación suicida. Según Fernández \& Merino (2001) en adolescentes existiría mayor prevalencia de ideación suicida en mujeres. La ideación suicida estaría mayormente asociada a depresión en mujeres y a un autoconcepto negativo en hombres (Fernández \& Merino, 2001).

La evidencia muestra que el riesgo suicida aumenta cuando existen ECNT, depresión o problemas emocionales, un entorno de violencia y abuso de sustancias principalmente (Nielsen, Wang \& Brille-Brahe, 1990; Frierson, 1991; Ajdacic-Gross et al., 2008; Lutz et al., 2016). Se estima que al menos un 16\% de las personas sin ECNT presenta ideación suicida en algún momento de su vida, mientras que un $25,2 \%$ de las personas con alguna ECNT lo haría, porcentaje que aumentaría a un $35 \%$ con la presencia dos o más enfermedades crónicas (Druss \& Pincus, 2000).

Existen modelos teóricos para la explicación del comportamiento suicida, varios de estos ponen énfasis en los motivos sociales que lo desencadenan: Emile Durkheim, señala que el suicidio depende del temperamento de sus ejecutores, por lo que debe existir un entorno favorable para su desarrollo, teniendo un rol fundamental la sociedad, la familia, la escuela, los grupos en que se participa (Durkeheim, 1897); Aaron T. Beck, propone un modelo cognitivo de estudio del suicidio asociándolo principalmente a la depresión y desesperanza (Beck, 1996; Beck, Kovacs \& Weissman, 1979; Wenzel \& Beck, 2008); Tomas Joiner propone la Teoría Interpersonal del Suicidio, desarrollando dianas terapéuticas sobre las que trabajar con las personas en riesgo de suicidio. La teoría relaciona el deseo suicida con la presencia de dos factores interpersonales: La falta de pertenencia y la sensación de ser una carga para los otros significativos. El tercer factor propuesto sería la capacidad adquirida de llevar a cabo el suicidio; las personas que sienten el deseo de suicidarse y además han desarrollado la capacidad de hacerlo serán las que lleven a cabo intentos de suicidio con mayor letalidad y tengan más posibilidades de morir por esa causa (Joiner, 2007). Por lo que finalmente, los tres modelos teóricos resaltan el rol social de la conducta suicida y la posibilidad de prevención desde el manejo de los factores ambientales y personas influyentes.

Dada la evidencia expuesta, el objetivo del presente estudio es identificar las asociaciones entre apoyo social percibido (ASP), riesgo suicida (RS) y presencia de ECNT en estudiantes universitarios. Nuestras hipótesis planteadas fueron:

1. Existe asociación entre el ASP y la presencia de ECNT en estudiantes universitarios.

2. Existe asociación entre el RS y la presencia de ECNT en estudiantes universitarios.

3. Existe asociación entre el ASP y el RS en estudiantes universitarios.

4. Existe asociación entre ASP y RS según presencia de ECNT en estudiantes universitarios.

\section{Método}

Diseño: Estudio no experimental de casos y controles, considerando que las variables no serán manipuladas. Participantes: La población la componen todos los estudiantes de la Universidad Autónoma de Chile, sede Temuco. La muestra fue obtenida por un muestreo no aleatorio intencionado. El criterio de inclusión de los sujetos del grupo de los casos fue: Tener diagnóstico de una ECNT mayor a un año. El criterio de exclusión del grupo de los casos fue: Tener más de una ECNT. Se realizó emparejamiento entre casos y controles por 
edad, género y facultad, asegurando que los grupos fueran homogéneos, considerando un control por cada caso. Logrando finalmente obtener 41 sujetos en cada grupo.

Procedimiento: Inicialmente, el protocolo de investigación fue presentado y aprobado por el Comité de Ética de la carrera de Psicología, de la Universidad Autónoma de Chile, sede Temuco ( $\left.\mathrm{n}^{\circ} 00012\right)$. Se realizaron dos encuestas, la primera se realizó en el periodo de clases previo consentimiento de los directores, profesores y alumnos, en donde se solicitaron los siguientes antecedentes: Nombre, edad, carrera, género, correo electrónico, enfermedad que padece y antigüedad de la enfermedad. Y la segunda encuesta se realizó previa firma de consentimiento informado, de forma individual, aplicando los instrumentos descritos a continuación, tanto en sujetos con y sin ECNT emparejados por edad ( $₫ 2$ años), facultad y sexo, por cada caso. Los participantes no recibieron ninguna recompensa por su participación.

En los casos en que estudiantes universitarios que presentaron RS, se envió una carta formal a su director de carrera y a la directora de la clínica Psicológica de la misma Universidad, con el nombre del sujeto en riesgo para dejar constancia, citar a entrevista y poder evaluar su riesgo suicida, tomando las medidas de resguardo correspondientes. Este protocolo de acción estaba explicitado en los consentimientos firmados previamente por los sujetos.

Instrumentos: Escala de Suicidalidad de Okasha (Okasha, Lotaif \& Sadek, 1981). Cuestionario de autoreporte diseñado para detectar RS sin necesidad de realizar un diagnóstico psiquiátrico. Fue validada en Chile para ser utilizada con adolescentes (Salvo, Melipillán \& Castro, 2009), presentando adecuadas propiedades psicométricas. La escala está constituida por cuatro ítems en formato Likert que se puntúan de 0 a 3 puntos para cada una (nunca, casi nunca, algunas veces, muchas veces). La sumatoria de los tres primeros ítems, genera el puntaje de RS, que puede variar entre 0 y 9 puntos. Mientras más alto, mayor RS. El ítem de intento de suicidio se considera aparte y se puntúa de 0 a 3 puntos según la cantidad de intentos: Ningún intento, un intento, dos intentos, tres o más intensos. Además, se considera que los sujetos presentan RS si tienen puntajes mayores o iguales a 5 (Salvo, Melipillán \& Castro; 2009).

Escala Multidimensional de la Percepción de Apoyo Social (MSPSS) (Zimet et al., 1988). Esta escala fue construida con la finalidad de medir niveles de apoyo percibido por los individuos en tres áreas: Familia, amigos y pareja u otros significativos. Está compuesta por 12 ítems en formato Likert cuyas opciones de respuesta van desde: 1 (muy en desacuerdo) a 7 (muy de acuerdo). Los puntajes más altos señalan mayor apoyo social percibido (Zimet, et al, 1988). La MSPSS fue traducida y adaptada para su uso en Chile por Arechabala \& Miranda (2002) y fue adaptada y validada en adolescentes, reduciendo la escala de frecuencia a 4 niveles, desde 1 (casi nunca) a 4 (casi siempre o siempre), así la escala va desde un puntaje mínimo de 12 a un máximo de 48 (Arechabala \& Miranda, 2002). La MSPSS es un instrumento de aplicación breve y simple de responder y ha demostrado características psicométricas adecuadas para ser utilizada en población adolescente (Arechabala \& Miranda, 2002).

Variables: Evento de interés, presencia de ECNT, variable de tipo nominal, con dos valores: Presente y Ausente. Variables de asociación: ASP, esta es una variable cuantitativa de razón, con valores que van de 12 a 48 puntos, en donde los valores más altos indican mayor ASP. Además, se consideran las tres subescalas: ASP Familiar (ASPF), ASP Amigos (ASPA) y ASP Persona Significativa (ASPPS). El RS, se evalúa de tres formas: 1) Nivel de Riesgo Suicida (NRS), variable cuantitativa de razón, con valores de 0 a 12 , en donde los valores altos indican mayor riesgo; 2) Presencia de Riesgo Suicida (PRS), variable dicotómica, sujetos con puntajes $\geq$ a 5 con RS, < a 5, sin RS; 3) Intento Suicida Previo (ISP), se considera si ha tenido o no intentos suicidas previos, como variable dicotómica, presencia y ausencia.

Análisis de datos: En primer lugar, para identificar la consistencia interna de las dos escalas administradas se realizaron análisis utilizando el coeficiente Alfa de Cronbach con cada instrumento en general y considerando sus subdimensiones.

Posteriormente se realizaron análisis exploratorios con las variables NRS y ASP, de forma general y segmentada por casos y controles, encontrando que no se distribuyen cercanos a la curva normal, ni en general, ni segmentada según presencia de enfermedad crónica. Considerando esto y el reducido número de sujetos, se decidió utilizar pruebas no paramétricas para identificar el cumplimiento de las hipótesis. Luego, se realizaron análisis descriptivos utilizando medianas y rangos con las variables NRS, ASP, ASPF, ASPA, ASPPS en general y segmentada según presencia de ECNT. En el caso de la variable ECNT, se describió utilizando frecuencias y porcentajes. 
Para identificar la asociación entre ASP y RS según presencia de ECNT en estudiantes universitarios, se utilizó prueba U de Mann Whitney con las variables cuantitativas y Exacto de Fischer con las variables dicotómicas.

Para poder identificar la relación entre ASP y RS en estudiantes universitarios, se realizó una correlación bivariada con el estadístico de rho de Spearman con las variables cuantitativas y U de Mann Whitney con las variables dicotómicas.

En relación con la última hipótesis esta se comprobó a través de una correlación parcial, pudiendo determinar si finalmente es la presencia de ECNT es la que determina la asociación entre ASP y RS.

Todos los análisis se realizaron con el programa estadístico SPSS, versión 20.0, con un intervalo de confianza del 95\%.

\section{Resultados}

La muestra está constituida por 82 sujetos, 41 de estos presentan al menos una ECNT. La mediana general de edad es de 22 años, 23 en los sujetos sin ECNT y 22 en los sujetos con ECNT, con rangos de 9, 8 y 8, respectivamente. En relación a la variable sexo, hay 66 sujetos de sexo femenino (36 en el grupo con ECNT y 30 en el grupo sin ECNT). De todos los participantes 56 pertenecen a la Facultad de Educación, Ciencias Sociales y Humanidades y 16 a la Facultad de Ciencias de la Salud, en el grupo con ECNT hay 27 sujetos en la Facultad de Educación, Ciencias Sociales y Humanidades y 9 sujetos en la Facultad de Ciencias de la Salud y en el grupo sin ECNT hay 29 sujetos en la Facultad de Educación, Ciencias Sociales y Humanidades y 7 sujetos en la Facultad de Ciencias de la Salud. Los análisis de comparación de grupos indican que no existen diferencias significativas entre estos, con valores $p>0,05$ en todos los casos, lo que indicaría homogeneidad entre los grupos (Ver Tabla 1).

Tabla 1. Características de la muestra

\begin{tabular}{|c|c|c|c|c|}
\hline & & Mdn & Rango & $p$ \\
\hline \multirow{3}{*}{ Edad } & General & 22 & 9 & - \\
\hline & Sin ECNT & 23 & 8 & \multirow{2}{*}{.230} \\
\hline & Con ECNT & 22 & 8 & \\
\hline \multirow{6}{*}{ Género } & \multirow{2}{*}{ General } & Masculino & $18,2 \%$ & \multirow{2}{*}{-} \\
\hline & & Femenino & $81,8 \%$ & \\
\hline & \multirow{2}{*}{ Sin ECNT } & Masculino & $26,8 \%$ & \multirow{4}{*}{.164} \\
\hline & & Femenino & $73,2 \%$ & \\
\hline & \multirow{2}{*}{ Con ECNT } & Masculino & $12,2 \%$ & \\
\hline & & Femenino & $87,8 \%$ & \\
\hline \multirow{9}{*}{ Facultad } & \multirow{3}{*}{ General } & Educ, Soc y Hum & $63,6 \%$ & \multirow{3}{*}{-} \\
\hline & & Salud & $25,0 \%$ & \\
\hline & & Ing y Const & $11,4 \%$ & \\
\hline & \multirow{4}{*}{ Sin ECNT } & Educ, Soc y Hum & $70,7 \%$ & \multirow{6}{*}{.851} \\
\hline & & Salud & $17,1 \%$ & \\
\hline & & Ing y Const & $12,2 \%$ & \\
\hline & & Educ, Soc y Hum & $65,9 \%$ & \\
\hline & \multirow[t]{2}{*}{ Con ECNT } & Salud & $22,0 \%$ & \\
\hline & & Ing y Const & $12,2 \%$ & \\
\hline
\end{tabular}

Los análisis de fiabilidad mostraron un valor de alfa de Cronbach para la escala de RS de 0,835 y en la escala de ASP un valor de 0,907, mostrando una buena consistencia interna inter-ítem. Sin recomendar la eliminación de ningún ítem para aumentar la consistencia en ninguna de las dos escalas. La fiabilidad en las sub dimensio- 
nes de ASP también es adecuada, con puntuaciones de 0,923 en ASPA, 0,885 en ASPF y 0,879 en ASPPS, sin recomendar eliminar ninguno de los ítems.

En relación con la PRS el 25\% de la muestra tiene riesgo y el 10,9\% de los sujetos ha tenido ISP. En el grupo de los sujetos con ECNT la PRS baja a 22\% y los ISP a 14,6\%. A diferencia del grupo sin ECNT en donde los porcentajes son de $24,4 \%$ y $4,9 \%$ respectivamente. La prueba estadística exacto de Fisher no entrega evidencia suficiente para señalar que estás diferencias de proporciones muestren asociación entre las variables con valores $p$ de 1 y 0,264 .

En relación con los análisis descriptivos del ASP podemos observar que no existen diferencias numéricas entre presencia de ECNT, por otra parte, la probabilidad de rechazar la hipótesis de nulidad es baja, por lo que los datos no entregan información suficiente para afirmar diferencias entre las medianas. Esta tendencia se repite al analizar cada una de las dimensiones del ASP (ver Tabla 2).

Con respecto al NRS sucede similar a lo mencionado anteriormente con el ASP, en donde tampoco existen diferencias ni numéricas, ni menos aun estadísticas que demuestren diferencias entre los dos grupos (ver Tabla 2).

Tabla 2. Análisis descriptivos y comparaciones de medianas de APS y NRS

\begin{tabular}{ccccc} 
& & Mdn & Rango & $p$ \\
\hline \multirow{3}{*}{ NRS } & General & 2.5 & 9 & - \\
& Sin ECNT & 2 & 8 & 0,795 \\
& Con ECNT & 2 & 9 & - \\
\multirow{3}{*}{ ASP } & General & 61.5 & 33 & 0,838 \\
& Sin ECNT & 62 & 33 & - \\
& Con ECNT & 62 & 30 & 0,855 \\
\hline \multirow{3}{*}{ ASPF } & General & 21 & 16 & - \\
& Sin ECNT & 21 & 16 & 0,612 \\
\hline \multirow{3}{*}{ ASPA } & Con ECNT & 20 & 15 & - \\
& General & 21 & 16 & 0.564 \\
\hline \multirow{3}{*}{ ASPPS } & Sin ECNT & 21 & 12 & 14 \\
& Con ECNT & 21 & 14 & 0 \\
& General & 23 & 10 & 0,564 \\
\hline
\end{tabular}

Al observar las medidas de ASP y sus subdimensiones, en relación con la presencia de RS e ISP observamos que los valores son mayoritariamente más altos siempre en ausencia de estos indicadores. En el caso del RS, la diferencia observada en el ASP, ASPF y ASPA es estadísticamente significativa. En los otros casos no hay probabilidad suficiente para rechazar la hipótesis de nulidad (ver Tabla 3). 
\& ZAYRA ANTÚNEZ

Tabla 3. Análisis descriptivos y comparaciones de medianas de APS, PRS y ISP

\begin{tabular}{|c|c|c|c|c|}
\hline & & Mdn & Rango & $p$ \\
\hline \multirow{2}{*}{ ASP } & Sin RS & 63 & 33 & \multirow{2}{*}{0,015} \\
\hline & Con RS & 57 & 33 & \\
\hline \multirow{2}{*}{ ASPF } & Sin RS & 21 & 16 & \multirow{2}{*}{$0,00 S$} \\
\hline & Con RS & 17 & 13 & \\
\hline \multirow{2}{*}{ ASPA } & Sin RS & 21 & 15 & \multirow{2}{*}{0,023} \\
\hline & Con RS & 19 & 16 & \\
\hline \multirow{2}{*}{ ASPPS } & Sin RS & 23 & 12 & \multirow{2}{*}{$0,26 \mathrm{C}$} \\
\hline & Con RS & 23 & 14 & \\
\hline \multirow{2}{*}{ ASP } & Sin ISP & 62 & 33 & \multirow{2}{*}{0,115} \\
\hline & Con ISP & 57 & 33 & \\
\hline \multirow{2}{*}{ ASPF } & Sin ISP & 21 & 16 & \multirow{2}{*}{0,053} \\
\hline & Con ISP & 18.5 & 14 & \\
\hline \multirow{2}{*}{ ASPA } & Sin ISP & 21 & 16 & \multirow{2}{*}{0,110} \\
\hline & Con ISP & 18 & 12 & \\
\hline \multirow{2}{*}{ ASPPS } & Sin ISP & 23 & 14 & \multirow{2}{*}{0,787} \\
\hline & Con ISP & 21.5 & 11 & \\
\hline
\end{tabular}

Al analizar la asociación entre el ASP y el NRS podemos observar que existe una relación significativa indirecta de baja intensidad $\left(r_{s}=-0,290 ; p<0,01\right)$, lo que da cuenta de que a mayor percepción de apoyo social menor el RS en los estudiantes universitarios. Así mismo sucede al analizar las sub dimensiones del ASP en donde en el caso de ASPF $\left(r_{s}=-0,332 ; p<0,005\right)$ y ASPA $\left(r_{s}=-0,269 ; p<0,05\right)$ se observa la misma tendencia, no así en el caso de ASPPS $(r=-0,102 ; p=0,332)$

Por otra parte, al analizar si la relación entre el ASP y el RS está determinada por la presencia de ECNT, se puede corroborar que independiente de la presencia de ECNT existe relación entre el ASP y el RS $(r=-0,325$; $p<0,01)$. Esto con un tamaño del efecto de 0,32 y una potencia estadística de $85,4 \%$. Así también se observa en el caso de las sub dimensiones del ASP en donde independiente de la influencia de ECNT sigue existiendo el mismo tipo de asociación ASPF $(r=-.0,329 ; p<.0,01)$, ASPA $(r=-0,299 ; p<0,01)$, ASPPS $(r=-0,197 ; p=0,08)$.

\section{Discusión}

El objetivo del presente trabajo fue identificar las asociaciones existentes entre ASP y el RS según presencia de ECNT en estudiantes universitarios. Dada la evidencia empírica que indica que tanto la depresión como la ideación y conducta suicida son frecuentes en adolescentes y adultos jóvenes con ECNT, siendo aproximadamente el doble que en jóvenes sin ECNT (Ferro, Rhodes, Kimber, Duncan, Boyle, Georgiades et al., 2017; Ali, Stone, Peters, Davies y Khunti, 2006; Fuller-Thomson y Sawyer 2009), se esperaba que nuestros hallazgos fueran similares, sin embargo, no encontramos diferencias estadísticamente significativas entre los jóvenes con y sin ECNT en términos de la asociación existente entre ASP y RS, lo cual indica que el apoyo social es un factor protector de ideación suicida tanto para jóvenes con ECNT como para jóvenes sin ECNT.

Nuestros resultados destacan la importancia del apoyo social, como un factor que podría generar una disminución en la ideación suicida, lo que coincide con lo planteado por autores como Farrell, Bolland y Cockerham (2015) y Bryant Miller, Esposito-Smythers y Leichtweis (2015), quienes plantean la relevancia de las redes de apoyo en los jóvenes con ECNT y como factor protector o de riesgo respecto al intento suicida. Así también lo confirman los estudios de Lamis et al. (2016) quienes plantean que el apoyo social funciona como un regulador de síntomas depresivos y pensamiento suicida. Específicamente, Hagedorn y Omar (2002) demuestran que el estrés causado por conflictos interpersonales (como conflictos familiares y con personas significativas) se relaciona con intentos suicidas en los jóvenes. Pérez, Ibáñez, Reyes, Atuesta \& Suárez (2008) encontraron que 
las personas jóvenes que mantenían relaciones disfuncionales con su familia y antecedentes de maltrato infantil presentaban más intentos suicidas.

En este sentido, cabe destacar el importante rol protector que desempeñan los factores sociales, ya que contar con relaciones sociales gratificantes, apoyo familiar e incluso, tener hijos pequeños (principalmente en las mujeres) potencian la capacidad de resistencia ante el intento suicida (OMS, 2014). En contraste, los jóvenes que no han intentado suicidarse reportan mayor unión y apoyo, así como también una mejor expresión de sus emociones dentro de su familia, acompañadas de una menor percepción de dificultades en ésta (Rivera, 2000). El apoyo social de familia y amigos es el que surge como más relevante para los jóvenes, por lo que las dificultades familiares también son las que provocan mayor aumento de estrés e ideación suicida (Feldman et al., 2008).

En el caso de las personas que padecen enfermedad crónica, estas se ven obligadas a modificar su estilo de vida, lo que implica generalmente que también su salud metal se vea afectada. Las enfermedades crónicas conllevan importante impacto emocional y secuelas psicológicas en el enfermo y su entorno (Abalo \& Jorge, 2016). En este sentido, se ha encontrado que el padecer una ECNT afecta con particular intensidad a los adolescentes y jóvenes, puesto que la desesperanza con respecto a la enfermedad puede impactarlos más fuertemente que a los adultos con más edad, lo que a su vez, se asocia con riesgo suicida (Conti, Mennitto, Di Francesco, Fraticelli, Vitacolonna \& Fulcheri, 2017; Radobuljac, Bratina, Battelino \& Tomori, 2009). Estos antecedentes son congruentes con nuestros hallazgos, donde la muestra con ECNT ha presentado intentos suicidas previos en un porcentaje que triplica (alrededor de un 15\%) a la muestra de jóvenes que no presenta ECNT (alrededor de un $5 \%$ ).

El suicidio es una de las principales prioridades de salud pública en todo el mundo, ya que en la actualidad es la segunda causa de muerte en los adolescentes y jóvenes de entre 15 y 29 años, siendo fundamental que la prevención incluya la identificación de grupos de alto riesgo (OMS, 2015b), como son los jóvenes y los enfermos crónicos. En este sentido, la población de la presente investigación es un grupo con un doble factor de riesgo, que presenta tres veces más intentos de suicidio, por lo que surge como un aspecto relevante la identificación del rol protector que tiene el apoyo social.

Por ende, la atención de salud mental a los jóvenes es prioritaria, puesto que es un grupo de alto riesgo susceptible a presentar problemas de salud mental debido a la etapa de transición y adaptación en que se encuentran (Brook \& Willoughby, 2015), pero es indispensable para los jóvenes con ECNT, sobre todo al considerar la alta prevalencia de ECNT en éste grupo de edad en la actualidad. Asimismo, la atención en salud mental no sólo debe centrarse en los síntomas, sino también en aspectos como la adaptación y afrontamiento ante la enfermedad, la preservación de la calidad de vida, la adherencia al tratamiento, entre otros (Abalo \& Jorge, 2016). Por ello, es importante que los estudiantes con ECNT puedan acceder a intervenciones integrales desde el inicio de la enfermedad hasta la estabilización de ésta.

Este estudio cuenta con una serie de limitaciones que hacen que sus hallazgos no sean generalizables. Entre ellas, se debe considerar que no se utilizó una muestra representativa de jóvenes, ya que, debido a la accesibilidad de la muestra, fueron seleccionados sólo estudiantes pertenecientes a la Universidad Autónoma de Chile, sede Temuco, lo que perjudicó poder comprobar las hipótesis 1 y 2 del estudio, contando con una alta probabilidad de cometer error tipo 2, por lo que es necesario tener un mayor número de sujetos, para identificar existen diferencias significativas entre el APS y el NRS según presencia de ECNT. Otra limitación importante es que no se abordaron otras variables que pudiesen influir en estos resultados tales como depresión, trastornos del estado del ánimo, trastornos de ansiedad, entre otros.

\section{Referencias}

Abalo, G. \& Jorge, A. (2016). Enfermedades crónicas no transmisibles: Un Abordaje desde los factores psicosociales. Saludy Sociedad, 7(2).

Adelman, R., Tmandova, L., Delgado, D., Dion, S., \& Lachs, M. (2014). Caregiven Burder: A Clinical Review, 311(10), 1052-1059. doi: 10.1001/jama.2014.304.

Ajdacic-Gross, V., Weiss, M., Ring, M., Hepp, U., Bopp, M., Gutzwiller, F., \& Rössler, W. (2008). Methods of suicide: international suicide patterns derived from the WHO mortality database. Bulletin of the World Health Organization, 86(9), 726-732. doi:10.2471/ BLT.07.043489 
Ali, S., Stone, M. A., Peters, J. L., Davies, M. J., \& Khunti, K. (2006). The prevalence of co-morbid depression in adults with type2 diabetes: a systematic review and meta-analysis. Diabetic Medicine, 23(11), 1165-73. doi:10.1111/j.1464-5491.2006.01943.x

Arechabala, M. C. \& Miranda, C. (2002). Validación de una escala de apoyo social percibido en un grupo de adultos mayores adscritos a un programa de hipertensión de la región metropolitana. Ciencia y Enfermería, 8(1), 49-55. doi: 10.4067/S0717-95532002000100007

Azevedo, L. F., Costa-Pereira, A., Mendonca, L., Dias, C. C., \& Castro-Lopes, J. M. The economic impact of chronic pain: a nationwide population-based cost-of-illness study in Portugal. European Journal of Health Economics, 17(1):87-98. doi: 10.1007/s10198-014-0659-4

Barra, E. (2004). Apoyo social, estrés y salud. Psicología y Salud, 14(2), 237-243.

Beck, A. T. (1996). Beyond belief: A theory of modes, personality, and psychopatology. In M. Salkovskis (Ed.), Frontiers of cognitive therapy (pp. 1-25). New York: The Guilford Press.

Beck, A. T., Kovacs, M., \& Weissman, A. (1979). Assessment of suicidal intention: The Scale for Suicide Ideation. Journal of Consulting and Clinical Psychology, 47(2), 343-352. doi: 10.1037/0022-006X.47.2.343

Brook, C. \& Willoughby, T. (2015). The Social Ties That Bind: Social Anxiety and Academic Achievement Across the University. Journal of Youth and Adolescence, 44(5), 1139-1152. doi: 10.1007/s10964-015-0262-8

Bryant Miller, A., Esposito-Smythers, C., \& Leichtweis, R. (2015). Role of Social Support in Adolescent Suicidal Ideation and Suicide Attempts, Journal of Adolescent Health, 56(3), 286-292. Recuperado de https://doi.org/10.1016/j.jadohealth.2014.10.265.

Canetto, S. S. \& Lester, D. (1995). Gender and the Primary Prevention of Suicide Mortality. Suicide and Life Threatening Behavior, 25(1), 58-69. doi: 10.1111/j.1943-278X.1995.tb00392.x

Conti, C., Mennitto, C., Di Francesco, G., Fraticelli, F., Vitacolonna, E., \& Fulcheri, M. (2017). Clinical Characteristics of Diabetes Mellitus and Suicide Risk. Frontiers in Psychiatry, 8, 40. doi:10.3389/fpsyt.2017.00040

Departamento de Estadísticas e Información de Salud (DEIS) del Ministerio de Salud de Chile (2016). Indicadores Básicos de Salud Chile 2016. Recuperado de http://www.deis.cl/wp-content/uploads/2018/12/IBS-2016.pdf

Díaz, R., Marulanda, F., \& Sáenz, X. (2009). Estudio epidemiológico del dolor crónico en Caldas, Colombia (Estudio Dolca). Acta Medica Colombiana, 34(3), 96-102.

Druss, B. \& Pincus, H. (2000). Suicidal ideation and suicide attempts in general medical illnesses. Archives of Internal Medicine, 160(10), 1522-1526. doi: 10.1001/archinte.160.10.1522

Durán, M. (2004). Las demandas sanitarias de las familias. Gaceta Sanitaria, 18(1), 195-200.

Durkheim, E. (1897). Le Suicide: Étude de sociologie. Paris. Félix Alcan.

Farrell C. T., Bolland J.M., \& Cockerham W. C. (2015). The role of social support and social context on the incidence of attempted suicide among adolescents living in extremely impoverished communities. Journal of Adolescent Health, 56(1), 59-65. doi: 10.1016/j. jadohealth.2014.08.015

Feldman, L., Goncalves, L., Chacon-Puignau, G., Zaragoza, J., Bagés, N., \& De Pablo, J. (2008). Relaciones entre estrés académico, apoyo social, salud mental y rendimiento académico en estudiantes universitarios venezolanos. Universitas Psychologica, 7(3), 739-752.

Fernández, N. \& Merino, H. (2001). Predictores de ideación suicida: Un estudio empírico en adolescentes. Revista de Psicopatología y Psicología Clínica, 6(2), 121-127. doi: 10.5944/rppc.vol.6.num.2.2001.3909

Ferro, M. A., Rhodes, A. E., Kimber, M., Duncan, L., Boyle, M. H., Georgiades, K., ... \& otros. (2017). Suicidal Behaviour Among Adolescents and Young Adults with Self-Reported Chronic Illness. The Canadian Journal of Psychiatry, 62(12), 845-853. doi: 10.1177 / 0706743717727242

Frierson, R. (1991). Suicide attempts by the old and the very old. Archives of Internal Medicine, 151(1), 141-144. doi:10.1001/archinte. 1991.00400010143022

Fuller-Thomson, E. \& Sawyer, J.L. (2009) Lifetime prevalence of suicidal ideation in a representative sample of Canadians with Type 1 diabetes. Diabetes Research and Clinical Practice, 83(1), 9-15. doi: 10.1016/j.diabres.2008.10.004

Garay-Sevilla, M. E. (2005) El mundo emocional del paciente diabético. II Encuentro Participación de la Mujer en la Ciencia. León, Guanajuato. Recuperado de http://octi.guanajuato.gob.mx/octigto/formularios/ideasConcyteg/Archivos/03022006_MUNDO_EMOCIONAL_PACIENTE_DIABETICO.pdf

Hagedorn, J. \& Omar, H. (2002). Retrospective analysis of youth evaluated for suicide attempt or suicidal ideation in an emergency room setting. International Journal of Adolescent Medicine and Health, 14(1), 55-60. doi: 10.1515/IJAMH.2002.14.1.55

Helgeson, V. S., Jakubiak, B., Van Vleet, M., \& Zajdel, M. (2018). Afrontamiento comunitario y adaptación a enfermedades crónicas: actualización teórica y evidencia. Revisión de Personalidad y Psicología Social, 22 (2), 170-195. doi: 10.1177/1088868317735767

Joiner, T. (2007). Why People Die by Suicide. Cambridge: Harvard University Press.

Kimberly, J. \& Serovich, J. (1999). The role of family and friend social support in reducing risk behaviors among HIV-positive gay men. AIDS Education and Prevention, 11(6), 465. 
Lamis, D. A., Ballard, E. D., May, A. M., \& Dvorak, R. D. (2016). Depressive symptoms and suicidal ideation in college students: the mediating and moderating roles of hopelessness, alcohol problems, and social support. Journal of Clinical Psychology, $72(9), 919$ - 939. doi: 10.1002/jclp.22295

Law, Y. W., Yip, P. S., Zhang, Y., \& Caine, E. D. (2014). The chronic impact of work on suicides and under-utilization of psychiatric and psychosocial services. Journal of affective disorders, 168, 254-261. doi: 10.1016/j.jad.2014.06.031

López, M. (2007). Ansiedad y depresión, reacciones emocionales frente a la enfermedad. Anales de Medicina Interna, 24(5), $209-211$.

López, T. M. T., Beltrán, C. A., Moreno, M. P., \& Estrada, J. G. S. (2007). Dimensiones conceptuales del bienestar de personas con padecimientos crónicos. Salus, 8(4).

Lutz, J., Morton, K., Turiano, N. A., \& Fiske, A. (2016). Health Conditions and Passive Suicidal Ideation in the Survey of Health, Ageing, and Retirement in Europe. The Journals of Gerontology Series B: Psychological Sciences and Social Sciences, 19. doi: 10.1093/ geronb/gbw019

Ministerio de Salud. (2013). Programa Nacional de Prevención del Suicidio: Orientaciones para su implementación, 21-37. Recuperado de http://web.minsal.cl/sites/default/files/Programa_Nacional_Prevencion.pdf.

Miranda, I., Cubillas, M. J., Román, R., \& Abril, E. (2009). Ideación suicida en población escolarizada infantil: Factores psicológicos asociados. Salud mental, 32, 495-502.

Navarro, D. A. (2007). Aspectos psicológicos y sociales de la Diabetes Mellitus. En: Navarro Despaigne DA. Diabetes Mellitus, Menopausia y Osteoporosis. La Habana: Editorial Científico-Técnica; 2007. p. 110-3.

Nielsen, B., Wang, A. G., \& Brille-Brahe, U. (1990). Attempted suicide in Denmark. IV. A five-year follow-up. Acta Psychiatrica Scandinavica, 81(3), 250-254. doi: 10.1111/j.1600-0447.1990.tb06490.x

Oblitas, L. (2006). Psicología de la Salud y Calidad de Vida. International Thomson Editores México D.F. (1) 27 - 35.

Okasha, A., Lotaif, F., \& Sadek, A. (1981). Prevalence of suicidal feelings in a sample of non-consulting medical students. Acta Psychiatrica Scandinavica, 63(5), 409-415. doi: 10.1111/j.1600-0447.1981.tb00690.x/full

Organización Mundial de la Salud (2014). Preventing suicide. A global imperative. Geneve: World Health Organization. Recuperado de https://www.who.int/mental_health/suicide-prevention/world_report_2014/en/

Organización Mundial de la Salud. (2015a). Enfermedades Cardiovasculares. Recuperado de http://www.who.int/mediacentre/factsheets/ fs $317 /$ es/

Organización Mundial de la Salud. (2015b). Datos y cifras sobre el suicidio: infografía; 2015. Recuperado de http://www.who.int/mental_health/suicide-prevention/infographic/es/

Pérez, J. \& Martin, F. (2006). El apoyo social. Barcelona/Madrid. Recuperado de: http://www.insht.es/InshtWeb/Contenidos/Documentacion/FichasTecnicas/NTP/Ficheros/401a500/ntp_439.pdf

Pérez, I., Ibáñez, M., Reyes, J., Atuesta, J., \& Suárez, M. (2008). Factores Asociados al Intento Suicida e Ideación Suicida Persistente en un Centro de Atención Primaria. Revista de Salud Pública, 10(3), 374-385.

Ponce, J. M., Velásquez, A., Márquez, E., López, L., \& Bellido, L. (2009). Influencia del apoyo social en el control de las personas con diabetes. Index de Enfermería: Información, 18(4): 224-228. doi: 10.4321/S1132-12962009000400002

Radobuljac, M. D., Bratina, N. U., Battelino, T., \& Tomori, M. (2009). Lifetime prevalence of suicidal and self-injurious behaviors in a representative cohort of Slovenian adolescents with type1 diabetes. Pediatric Diabetes, 10(7), 424-431. Recuperado de: https://doi. org/10.1111/j.1399-5448.2009.00501.x

Rivera, M. E. (2000). Percepción de las relaciones intrafamiliares y su relación con el intento suicida en adolescentes. La Psicología Social en México, 8, 555-559.

Rivera-Heredia, M. \& Andrade-Palos, P. (2006). Recursos individuales y familiares que protegen al adolescente del intento suicida. Revista Intercontinental de Psicología y Educación, 8(2), 23-40.

Salvo, L., Melipillán, R., \& Castro, A. (2009). Confiabilidad, validez y punto de corte para escala de screening de suicidalidad en adolescentes. Revista Chilena de Neuro-Psiquiatría, 47(1), 16-23. doi: 10.4067/S0717-92272009000100003

Sánchez, R., Cáceres, H., \& Gómez, D. (2002). Ideación suicida en adolescentes universitarios: prevalencia y factores asociados. Biomédica, 22, 407-416. doi: 10.7705/biomedica.v22iSupp2.1189

Schofield, D., Shrestha, R. N., Cunich, M. M., Tanton, R., Veerman, L., Kelly, S. J., \& Passey, M. E. (2016). Economic costs of chronic disease through lost productive life years (PLYs) among Australians aged 45-64 years from 2015 to 2030: results from a microsimulation model. BMJ Open, 6(9), e011151. doi:10.1136/bmjopen-2016-011151

Serrano González, M. I. (2002). Educación para la salud del siglo XXI: comunicación y salud. En Educación para la salud del siglo XXI: Comunicación y salud. Alezeia.

Souza, L. D. d. M., Silva, R. A. d., Jansen, K., Kuhn, R. P., Horta, B. L., \& Pinheiro, R. T. (2010). Suicidal ideation in adolescents aged 11 to 15 years: prevalence and associated factors. Revista Brasileira de Psiquiatría, 32, 37-41. doi: 10.1590/S1516-44462009005000011 
Tunbridge, W. M., Evered, D. C., Hall, R., Appleton, D., Brewis, M., Clark, F., ... \& otros. (1977). The spectrum of thyroid disease in a community: the Whickham survey. Clinical endocrinology, 7(6), 481-493. doi:10.1111/j.1365-2265.1977.tb01340.x

Vega Angarita, O. M. \& González Escobar, D. S. (2009). Apoyo social: Elemento Clave en el Afrontamiento de la Enfermedad crónica. Enfermería Global, 16, 0-0.

Vinaccia, S. \& Orozco, L. M. (2005). Aspectos psicosociales asociados con la calidad de vida de personas con enfermedades crónicas. Diversitas, 1(2), 125-137.

Wenzel, A. \& Beck, AT. (2008). A cognitive model of suicidal behavior: theory and treatment. Applied \& Preventive Psychology: Journal of the American Association of Applied and Preventive Psychology, 12, 189-201. doi: 10.1016/j.appsy.2008.05.001

Yeo, M. \& Sawyer, S. (2005). Chronic illness and disability. British Medical Journal, 330, 721-723. doi: 10.4067/S0717-92272009000100003

Zimet, G. D., Dahlem, N. W., Zimet, S. G., \& Farley, G. K. (1988). The Multidimensional Scale of Perceived Social Support. Journal of Personality Assessment, 52(1), 30-41. doi: 10.1207/s15327752jpa5201_2 